\title{
Edulcorantes: sob a ótica dos consumidores
}

\author{
Sweeteners: from the view point of consumers \\ Edulcorantes: desde el punto de vista de los consumidores
}

Recebido: 04/02/2022 | Revisado: 10/02/2022 | Aceito: 22/02/2022 | Publicado: 03/03/2022

\author{
Paula Caroline dos Santos Gomes \\ ORCID: https://orcid.org/0000-0002-6711-952X \\ Universidade Federal de Sergipe, Brasil \\ E-mail:paulacarolinesgomes@gmail.com \\ Tatiana Pacheco Nunes \\ ORCID: https://orcid.org/0000-0001-9106-8622 \\ Universidade Federal de Sergipe, Brasil \\ E-mail: tpnunes@uol.com.br \\ Antônio Martins de Oliveira Junior \\ ORCID: https://orcid.org/0000-0002-8635-7048 \\ Universidade Federal de Sergipe, Brasil \\ E-mail: amartins.junior@gmail.com
}

\begin{abstract}
Resumo
Os adoçantes ou edulcorantes, são substâncias químicas com capacidade de substituir total ou parcialmente o açúcar, primeiramente formulados para atender a população diabética, atualmente estão sendo cada vez mais utilizados para reduzir e controlar o consumo de calorias pela população. O objetivo deste trabalho foi avaliar a percepção dos consumidores sobre o consumo de edulcorantes e determinar os valores de ingestão diária desse aditivo pelos participantes. A metodologia do trabalho consistiu na aplicação de um questionário via Google Forms com perguntas relativas a dados demográficos e sobre o perfil do consumidor de alimentos com edulcorantes, como o motivo do consumo, a presença de patologia, produtos consumidos, a frequência de consumo, a quantidade ingerida e o peso corpóreo. Os dados coletados foram tabulados no Microsoft Office Excel 2013. Verificou-se que o principal motivo do consumo de produtos diet/light e adoçantes de mesa líquidos e sachês é a preocupação com a saúde, também foi observado que a maioria (51\%) alegou conhecer o adoçante utilizado na formulação dos produtos consumidos e que a ingestão de produtos diet/light foi diretamente proporcional à escolaridade e renda dos participantes. Por último e não menos importante, também foi verificado que nenhum dos participantes ultrapassou o índice de ingestão diária aceitável, sendo assim, o consumo desses produtos não representou risco toxicológico ao grupo estudado.
\end{abstract}

Palavras-chave: Edulcorantes; Consumidor; Ingestão diária aceitável.

\begin{abstract}
The sweeteners are chemical substances with the capacity to replace all or part of sugar, first formulated to meet the diabetic population, are currently being used to reduce and control calorie intake by the population. The objective of this study was to evaluate the perception of consumers about the consumption of sweeteners and to determine the daily intake values of this additive by the participants. The methodology of this study consisted of applying a questionnaire via Google Forms regarding demographic data and the profile of consumers of food with sweeteners, such as the reason for consumption, the presença of pathologies, products consumed, frequency of consumption, amount ingested, and body weight. The collected data were tabulated in Microsoft Office Excel 2013. It was found that the main reason for the consumption of diet/light products and liquid tabletop sweeteners and sachets is the concern with health, it was also observed that the majority $(51 \%)$ claimed to know the sweetener used in formation of the products consumed and that the intake of diet/light products was directly proporcional to the education and income of the participants. Last but not least, it was also verified that none of the participants exceeded the acceptable daily intake index, thus the consumption of these products did not pose any toxicological risk to the group studied.
\end{abstract}

Keywords: Sweeteners; Consumer; Acceptable daily intake.

\section{Resumen}

Los edulcorantes, son sustancias químicas con la capacidad de sustituir total o parcialmente al azúcar, formulados por primera vez para satisfacer a la población diabética, actualmente se utilizan cada vez más para reducir y controlar la ingesta de calorías por parte de la población. El objetivo de este estudio fue evaluar la percepción de los consumidores sobre el consumo de edulcorantes y determinar los valores de ingesta diaria de este aditivo por parte de los participantes. La metodología del trabajo consistió en la aplicación de un cuestionario a través de Google Forms con preguntas relativas a datos demográficos y al perfil del consumidor de alimentos con edulcorantes, como el motivo de consumo, la presencia de patología, los productos consumidos, la frecuencia de consumo, la cantidad 
ingerida y el peso corporal. Los datos recogidos se tabularon en Microsoft Office Excel 2013. Se comprobó que la principal razón para el consumo de productos dietéticos/light y edulcorantes líquidos de mesa y de sobremesa es la preocupación por la salud, también se observó que la mayoría (51\%) afirmó conocer el edulcorante utilizado en la formulación de los productos consumidos y que la ingesta de productos dietéticos/light era directamente proporcional a la educación y los ingresos de los participantes. Por último, pero no menos importante, también se comprobó que ninguno de los participantes superó el índice de ingesta diaria aceptable, por lo que el consumo de estos productos no supuso ningún riesgo toxicológico para el grupo estudiado.

Palabras clave: Edulcorantes; Consumidor; Ingesta diaria aceptable.

\section{Introdução}

Os adoçantes ou edulcorantes, são substâncias químicas, obtidas de matérias-primas naturais ou artificiais, desenvolvidas pela indústria de alimentos, com o poder de substituir total ou parcialmente o açúcar, sem acrescentar calorias à preparação (Barros et al., 2021).

Inicialmente formulados para atender às necessidades da população diabética, atualmente os adoçantes estão sendo cada vez mais utilizados como forma de reduzir e controlar o consumo de calorias pela população. Condições como preocupação com o corpo e à saúde contribuem para a crescente utilização e comercialização desses produtos (Barros et al., 2021).

Há uma grande variedade de adoçantes utilizados na alimentação, cada um com doçura individual, características tecnológicas e Ingestão Diária Aceitável (IDA) (Honório, et al., 2021). Os edulcorantes permitidos pela legislação brasileira podem ser divididos em dois tipos: os edulcorantes naturais, que são substâncias orgânicas encontradas na natureza com capacidade de conferir sabor doce aos alimentos e os edulcorantes sintéticos, que são os obtidos em processos químicos equivalente sãos de origem natural e também podem ser classificados de acordo com sua função, em nutritivos e não nutritivos (Oliveira, et al, 2019).Os adoçantes não nutritivos incluem a sacarina, aspartame, Acessulfamek, sucralose, neotame, alitame, neoesferidina, taumatina, ciclamato e stévia. e os nutritivos são: sacarose, frutose, glicoseepolióis (Lopes, et al., 2020).

Os adoçantes artificiais, também conhecidos como substitutos do açúcar ou adoçantes sem açúcar, são substâncias sintéticas utilizadas para substituir o açúcar durante o processo de adoçamento de diversos produtos. Esses aditivos são identificados como adoçantes intensivos porque exibem um poder adoçante maior do que o açúcar convencional (Muñoz, et al., 2022).

O uso de aditivos edulcorantes em alimentos, bem como os seus respectivos limites máximos, deve atender a Resolução da Diretoria Colegiada (RDC) n 18, de 24 de março de 2008.Os edulcorantes somente podem ser utilizados para substituição total ou parcial de açúcar em alimentos e bebidas para controle de peso, alimentos para dietas restritas ou controladas de açúcares, com informação nutricional complementar, referente aos atributos "não contém açúcares", "sem adição de açúcares”, "baixo em açúcares "ou“ reduzido em açúcares” ou, ainda, referente aos atributos "baixo em valor energético" ou "reduzido em valor energético", quando é feita a substituição parcial ou total do açúcar (Brasil, 2008).

A substituição parcial/total do açúcar por adoçantes em bebidas industrializadas é uma tendência mundial, além de ser principal estratégia da indústria de bebidas para atender à recomendação de redução de açúcar da Organização Mundial da Saúde (OMS), sem rejeição das bebidas pelos consumidores(Honorio, et al. 2021).

Portanto, a cada dia o consumo de adoçantes se torna mais massivo em nossa sociedade e eles estão presentes em uma grande variedade de alimentos. Os adoçantes mantêm a palatabilidade da dieta, permitindo que eles substituam o açúcar em muitos alimentos e, assim, reduzam sua ingestão calórica. A ingestão é segura, mas é necessário determinar a quantidade de adoçantes nos alimentos e monitorar o consumo pela população, para determinar se estão ou não ultrapassando a IDA (Duran et al., 2013). Dessa forma, o presente trabalho tem por objetivo principal avaliar a percepção dos consumidores sobre o consumo de edulcorantes e determinar os valores de ingestão diária desse aditivo pelos participantes. 


\section{Metodologia}

Foi realizado um estudo descritivo quantitativo, no qual foi aplicado um formulário com utilização da plataforma Google Forms para coleta de dados. Na primeira parte do questionário foram coletadas informações relativas a dados demográficos, com perguntas abertas sobre a idade, o gênero e o estado brasileiro no qual o respondente estava morando e perguntas fechadas sobre a rendar familiar (menor que 1 salário mínimo, de 1 a 2 salários mínimos, de 3 a 4 salários mínimos, de 5 a 6 salários mínimos e acima de 6 salários mínimos) e sobre o grau de escolaridade (ensino fundamental incompleto, ensino fundamental completo, ensino médio incompleto, ensino médio completo, ensino superior incompleto e ensino superior completo). Na segunda parte foram coletadas informações sobre o perfil do consumidor de alimentos com edulcorantes onde se questionou por meio de perguntas fechadas, o motivo do consumo (preferência em relação ao açúcar, indicação médica ou nutricionista e preocupação com a estética), a presença de patologia (diabetes, hipertensão e ausência de patologia). Sobre o conhecimento dos edulcorantes contidos nos produtos consumidos e sobre a presença de sabor residual nesses produtos, os respondentes tinham como opção de resposta as alternativas sim e não. Ressalta-se que para as perguntas relacionadas ao motivo de consumo e a presença de patologia, os participantes, além das opções previamente descritas, também poderiam marcar a alternativa "outros", na qual podiam escrever abertamente sobre o assunto.

$\mathrm{Na}$ terceira parte do questionário, os participantes foram inqueridos em perguntas abertas sobre as marcas de chás prontos e refrigerantes diet/light consumidas e frequência de consumo, e existiam dentre as opções de resposta: uma vez ao dia, uma vez na semana e uma vez ao mês e caso consumissem mais que uma vez ao dia, semana ou mês, poderia ser indicado na opção "outros". Quanto à quantidade ingerida a cada consumo, as alternativas existentes no questionário eram: um copo americano $(200 \mathrm{~mL})$, uma lata $(350 \mathrm{~mL}), 1 \mathrm{~L}$ e novamente a opção outros. Também em pergunta aberta os participantes responderam sobre a marca dos adoçantes líquidos e dos adoçantes em saches consumidas, assim como a quantidade de gotas e/ou sachês que eram utilizados para adoçar as bebidas (cafés, chás e sucos). Por fim foi perguntado o peso corpóreo de cada participante.

Os dados coletados foram tabulados no Microsoft Office Excel 2013, e os resultados expressos em porcentagem por meio de tabelas e gráficos. Para estimar a Ingestão Diária Aceitável de cada participante, consultou-se a formulação das bebidas citadas pelos consumidores através da análise dos rótulos desses produtos e posteriormente foi realizado o cálculo com base no peso corpóreo do participante e com o limite de ingestão diária aceitável estabelecido para cada edulcorante pelo Joint Expert Committeeon Food Additives (JECFA).

A realização desta pesquisa foi aprovada pelo Comitê de Ética em Pesquisa da Universidade Federal de Sergipe (5.253.293). O Termo de Consentimento Livre Esclarecido (TCLE) foi apresentado aos participantes antes do questionário.

\section{Resultados e Discussão}

Um total de 123 indivíduos responderam à pesquisa por meio de formulário no google forms, dentre as respostas foram descartados 25 questionários devido a informações inconsistentes. As respostas se concentraram na região nordeste com $60 \%(n=58)$ seguido da região sudeste com $30 \%(n=30)$. As mulheres corresponderam a maior parte dos entrevistados, representando 64\% $(\mathrm{n}=63)$. Quanto à renda familiar, 32\% $(\mathrm{n}=31)$ dos participantes tinham renda acima de 6 salários mínimos, 13\% (n=13) renda entre 5 e 6 salários mínimos, 20\% (n=20) com renda de 3 a 4 salários mínimos. Os respondentes que tinham renda familiar entre 1 a 2 salários mínimos e menor que 1 salário mínimo foram 26\% (n=25) e 9\% (n=9), respectivamente. Observou-se que a maioria dos participantes possuía ensino superior completo com $60 \%$ ( $\mathrm{n}=58$ ), seguido superior incompleto com $25 \%(\mathrm{n}=25)$, caracterizando alto grau de escolaridade entre os entrevistados. Em relação à idade, 49\% ( $n=47)$ situavam-se entre 19 e 29 anos, seguido da faixa etária entre 30 a 49 anos, com 20\% ( $n=14)$. 
Sylvetsky e Rother, (2016) relataram em sua pesquisa que a prevalência de consumo de edulcorantes está associada às famílias de renda mais alta e maior escolaridade, assim como a maior tendência em consumo entre o sexo feminino, dados que corroboram com os encontrados na presente pesquisa.

Verificou-se que o adoçante de mesa líquido foi o mais consumido entre os entrevistados (64\%, n=63) e o consumo de adoçantes em sachês foi relatado por 20\% $(n=20)$. Quanto ao consumo dos produtos light/diet, os refrigerantes foram consumidos por $63 \%(\mathrm{n}=62)$ dos respondentes $(\mathrm{n}=26)$, seguido dos chás prontos, que foram lembrados por $27 \%$ dos consumidores $(\mathrm{n}=26)$, também foi observado que 39\% $(\mathrm{n}=39)$ consomem simultaneamente refrigerante diet/light e adoçante de mesa líquido, assim como 16\% ( $\mathrm{n}=16)$ consomem em conjunto refrigerantes e chás prontos diet/light.

Na Figura 1 são apresentados os motivos que levam os participantes a consumir produtos dietéticos ou com baixo teor de açúcar, e verificou-se que o maior percentual (31,5\%) respondeu preocupação com a saúde, seguido de preferência em relação ao açúcar com 25,2\% ( $\mathrm{n}=32)$. Esses dados diferem dos reportados por Bianchi (2012), onde há alguns anos, o principal motivo era a preferência em relação ao açúcar. Observou-se também que 8,7\% (n=9) dos entrevistados informaram que o consumo desses produtos está relacionado à vontade em reduzir a quantidade de açúcar ingerido, ou à facilidade e conveniência desses produtos, ou então devido à presença de pessoas com diabetes na família.

Quando questionados sobre a presença de alguma patologia (Figura 2), 70,48\% ( $\mathrm{n}=74)$ responderam que não possuíam nenhuma patologia, embora, na Figura 1, a saúde tenha sido o principal motivo da escolha de produtos diet/light em relação aos convencionais. Ademais, é possível observar que sete dos 98 respondentes $(6,67 \%)$ marcaram a opção "outros" em relação à presença de patologias e esses declararam ter enxaqueca, obesidade, altas taxas de triglicérides, enfisema pulmonar, artrite reumatoide, pré-diabetes, asma e condropatia. Hernandez, et al. (2019) mencionaram em seu trabalho que o aumento da ingestão de adoçantes em pessoas saudáveis se deve a mudanças no estilo de vida em todo mundo, especialmente em países com dieta ocidental.

Figura 1. Motivo de consumo de adoçantes e produtos diet/light. Figura 2. Porcentagem de presença de patologia.

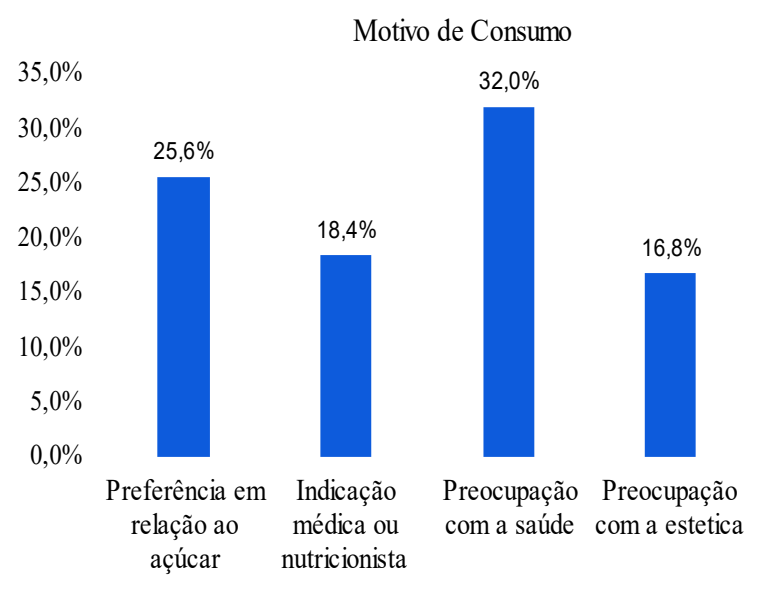

Fonte: Autores (2022).

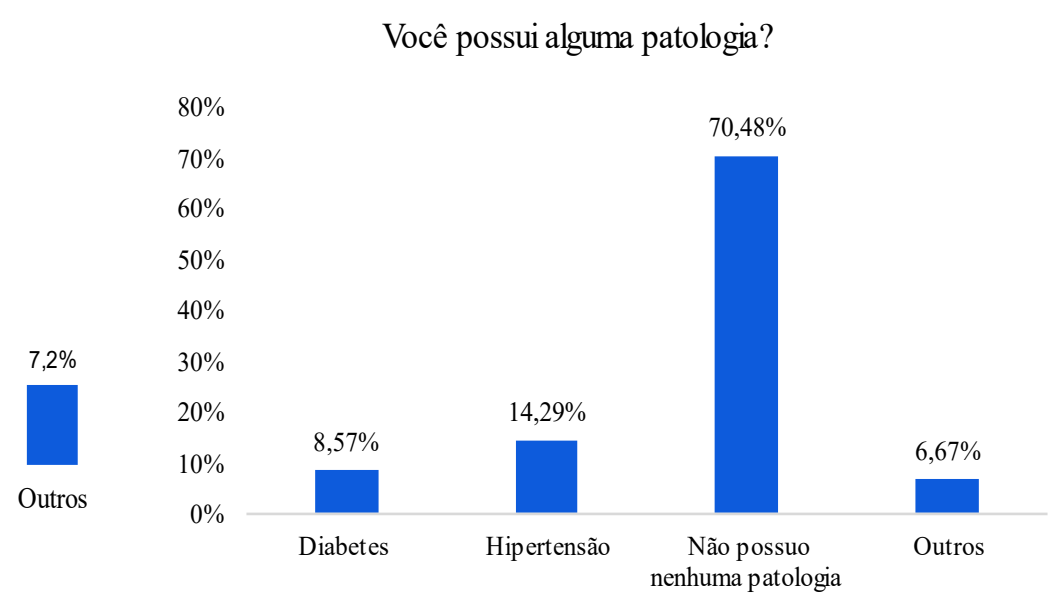

Fonte: Autores (2022)

Quando questionados sobre os edulcorantes presentes na formulação dos produtos consumidos, $51 \%$ ( $\mathrm{n}=50$ ) responderam que sabiam, enquanto $49 \%(\mathrm{n}=48)$ afirmaram desconhecer o edulcorante. Em relação à percepção de sabor residual, 66\% ( $\mathrm{n}=65$ ) afirmaram que sentem esse sabor, embora isso não afete no consumo desses produtos.

Em relação ao consumo de refrigerantes, observou-se que a Coca-Cola zero foi o mais citado entre os entrevistados (56\%, $\mathrm{n}=52)$, seguido de Guaraná Antártica Zero com 20\% (n=19), Sprite Zero com 5\%(n=4), Pepsi e Pepsi Black com 8\% e 2\%, respectivamente, Soda Reduzida em Açúcares com 2\% ( $\mathrm{n}=2)$ e Pepsi Twist Zero com 1\% (n=1). A Tabela 1ilustra os 
refrigerantes mais consumidos na presente pesquisa e o teor de edulcorantes presentes nessas formulações, de acordo com os rótulos desses produtos. É possível observar a associação entre diferentes edulcorantes na mesma bebida e isso deve-se ao fato da ação sinérgica que pode acontecer entre os edulcorantes.

Tabela 1. Quantidade de edulcorantes contidos nos refrigerantes consumidos pelos participantes de acordo com os rótulos.

\begin{tabular}{|c|c|}
\hline Refrigerante & Edulcorantes \\
\hline \multirow{3}{*}{ Coca-Cola zero } & Ciclamato de sódio $(27 \mathrm{mg} / 100 \mathrm{~mL})$ \\
\hline & Acessulfame de Potássio (15 mg/100 mL) \\
\hline & Aspartame $(12 \mathrm{mg} / 100 \mathrm{~mL})$ \\
\hline \multirow{2}{*}{ Fanta Zero } & Ciclamato de sódio $(70 \mathrm{mg} / 100 \mathrm{~mL})$ \\
\hline & Sacarina sódica (8 mg/100 mL) \\
\hline \multirow{2}{*}{ Guaraná Antártica Zero } & Aspartame $(34,8 \mathrm{mg} / 100 \mathrm{~mL})$ \\
\hline & Acessulfame de Potássio $(9 \mathrm{mg} / 100 \mathrm{~mL})$ \\
\hline \multirow{2}{*}{$\mathrm{H} 2 \mathrm{OH}$} & Aspartame $(31,5 \mathrm{mg} / 100 \mathrm{~mL})$ \\
\hline & Acessulfame de Potássio $(8,1 \mathrm{mg} / \mathrm{mL})$ \\
\hline \multirow{2}{*}{ Pepsi Black } & Sucralose $(18 \mathrm{mg} / 100 \mathrm{~mL})$ \\
\hline & Acessulfame de Potássio (11,2 mg/100 mL) \\
\hline \multirow{2}{*}{ Pepsi Twist Zero } & Aspartame $(35 \mathrm{mg} / 100 \mathrm{~mL})$ \\
\hline & Acessulfame de Potássio $(9 \mathrm{mg} / 100 \mathrm{~mL})$ \\
\hline \multirow{2}{*}{ Soda reduzido em Açucares } & Acessulfame de Potássio $(6,2 \mathrm{mg} / 100 \mathrm{~mL})$ \\
\hline & Sucralose (1,6 mg/100 mL) \\
\hline \multirow{2}{*}{ Soda Zero } & Acessulfame de Potássio $(9 \mathrm{mg} / 100 \mathrm{~mL})$ \\
\hline & Aspartame $(34,8 \mathrm{mg} / 100 \mathrm{ml})$ \\
\hline \multirow{2}{*}{ Sprite Zero } & Ciclamato de sódio $(72 \mathrm{mg} / 100 \mathrm{ml})$ \\
\hline & Sacarina sódica (11 mg/100 ml) \\
\hline
\end{tabular}

Fonte: Autores (2022).

A combinação entre o acessulfame e o aspartame é uma das principais associações utilizadas pela indústria de alimentos para adoçar refrigerantes, pois o elevado sabor residual característico do acessulfame é minimizado quando combinado a outros edulcorantes como aspartame, além disso, essa combinação compensa a perda de doçura causada pela degradação do aspartame durante o armazenamento (Lima, 2020).

Ciclamato associado à sacarina também está presente nas formulações de refrigerantes, e dentre os motivos dessa combinação relata-se a redução do sabor residual e o aumento do poder adoçante do ciclamato quando associado à sacarina. Portanto, a sacarina e o ciclamato são sinérgicos, permitindo a economia de custos para indústria através da redução da utilização das suas concentrações (Lima, 2020).

Também é notável a prevalência do uso de edulcorantes artificiais em todas as marcas de refrigerantes consumidos pelos participantes, essa predominância também foi relatada por Oliveira, et al. (2019), que analisaram diferentes rótulos de produtos diet/light em Teresina- Piauí.

Nas Figuras 3 e 4 são apresentadas a frequência de consumo dos refrigerantes diet/light e a quantidade ingerida a cada consumo, respectivamente. Pode-se observar que a maioria dos participantes consome esse tipo de bebida apenas uma vez na semana $(36 \%, \mathrm{n}=22)$, seguido de uma vez ao mês $(31 \%, \mathrm{n}=19)$, sendo a lata $(350 \mathrm{~mL})$ o volume máximo ingerido a cada consumo (58\%, n=35), como demonstrado na Figura 4. 
Figura 3. Frequência de consumo de refrigerantes diet/light. Figura 4. Quantidade de refrigerante diet/light ingerida.

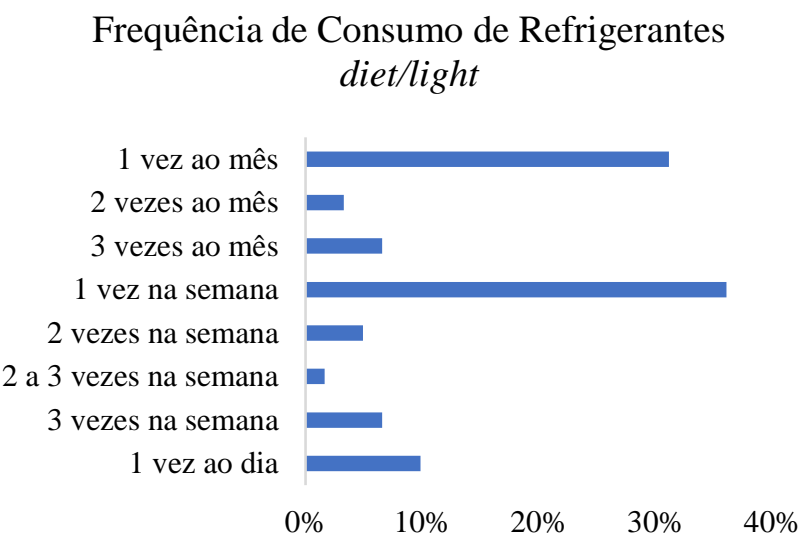

Fonte: Autores (2022).

\section{Quantidade de refrigerante diet/light ingerida a} cada consumo

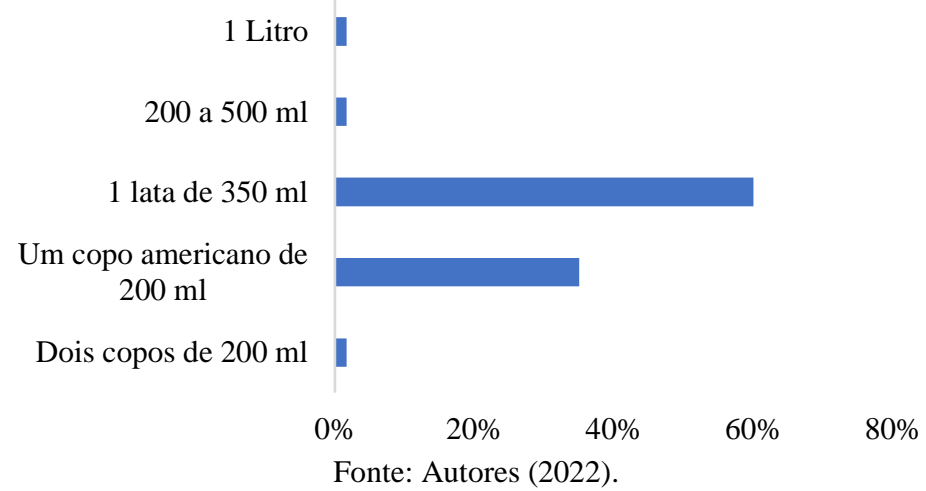

Em relação aos chás prontos citados pelos respondentes, verifica-se na Tabela 2, a combinação dos edulcorantes presentes nas formulações dessas bebidas.

Tabela 2. Edulcorantes contidos nos chás prontos diet/light consumidos pelos participantes.

\begin{tabular}{c|l}
\hline \multicolumn{1}{c|}{ Chás prontos diet/light } & \multicolumn{1}{c}{ Edulcorantes } \\
\hline \multirow{2}{*}{ Ice Tea Leão - Chá matte leão zero } & Sacarina de Sódio $(13 \mathrm{mg} / 100 \mathrm{ml})$ \\
& Acessulfame de Potássio $(6 \mathrm{mg} / 100 \mathrm{ml})$ \\
\hline Lipton ice zero açúcar pêssego & Sucralose $(5 \mathrm{mg} / 100 \mathrm{ml})$ \\
\hline
\end{tabular}

Fonte: Autores (2022).

Dentre as respostas obtidas, o chá pronto Ice Tea Leão foi o mais relatado com $79 \%(\mathrm{n}=23)$. Apenas um respondente informou o consumo de chás prontos da marca Feel Good e Natural, mas não informou o sabor. Como a formulação dos edulcorantes varia em função do sabor do produto, essa resposta não pode ser comutada.

Em relação à frequência de consumo de chás prontos e a quantidade ingerida em cada consumo (Figuras 5 e 6 , respectivamente), verificou-se que os consumidores bebem menos chás que refrigerantes, visto que a maioria (48\%, $\mathrm{n}=14$ ) respondeu consumir uma vez ao mês, diferente dos refrigerantes onde a maioria consumiu uma vez por semana. Além disso, também é possível verificar que a maioria $(86 \%, \mathrm{n}=19)$ ingere aproximadamente $200 \mathrm{~mL}$ por consumo, enquanto aqueles respondentes, que preferem refrigerante, bebem um volume maior $(350 \mathrm{~mL})$. Isso pode ser justificado principalmente pelo volume das embalagens normalmente comercializadas, bem como pela disponibilidade de oferta dessas bebidas no mercado. 
Figura 5. Frequência de consumo de chás prontos.

Frequência de Consumo de Chás prontos diet/light

3 vezes ao ano

Umvezao mês

Uma vezna semana

Uma vez ao dia

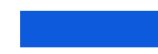

$0 \% \quad 10 \% \quad 20 \% \quad 30 \% \quad 40 \% \quad 50 \% \quad 60 \%$ Fonte: Autores (2022)
Figura 6. Quantidade ingerida de chás prontos a cada consumo.

Quantidade de chá pronto diet/light ingerida a cada consumo

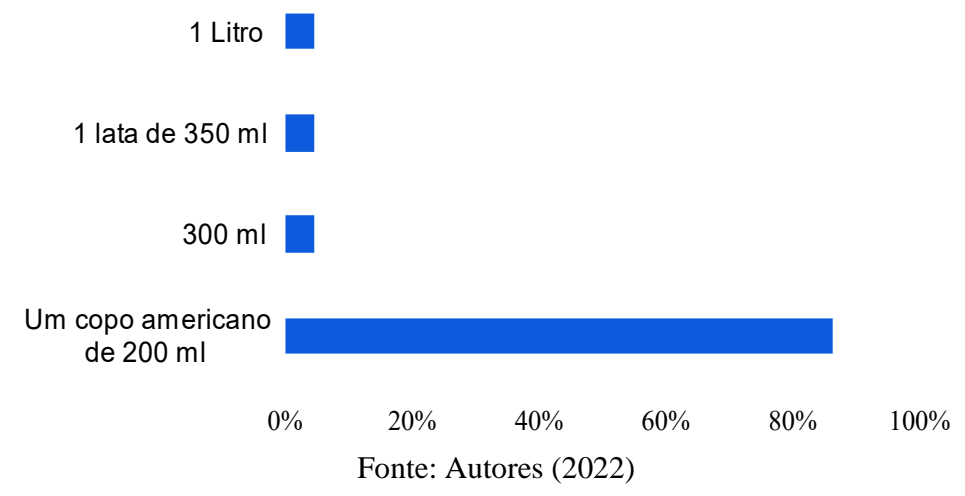

Fonte: Autores (2022)

A partir das respostas sobre a frequência de consumo, a quantidade ingerida dos refrigerantes e chás prontos diet/light consumidos e do peso corpóreo foi possível calcular o IDA de edulcorantes pelos participantes. Na Tabela a seguir (Tabela 3) é apresentada o IDA de cada edulcorante presente nas bebidas consumidas pelos participantes.

Tabela 3. Valor da Ingestão Diária Aceitável dos edulcorantes presentes nas formulações dos refrigerantes, chás e adoçantes de mesa estabelecidos pelo JECFA.

\begin{tabular}{c|c}
\hline Edulcorante & Ingestão Diária Aceitável (IDA) \\
\hline Acessulfame de potássio & $15 \mathrm{mg} / \mathrm{kg}$ de peso corpóreo \\
\hline Aspartame & $40 \mathrm{mg} / \mathrm{kg}$ de peso corpóreo \\
\hline Ciclamato de sódio & $11 \mathrm{mg} / \mathrm{kg}$ de peso corpóreo \\
\hline Glicosídeos de esteviol & $4 \mathrm{mg} / \mathrm{kg}$ de peso corpóreo \\
\hline Sacarina sódica & $5 \mathrm{mg} / \mathrm{kg}$ de peso corpóreo \\
\hline Sucralose & $15 \mathrm{mg} / \mathrm{kg}$ de peso corpóreo \\
\hline
\end{tabular}

Fonte: Joint Expert Committee on Food Additives(JECFA)

Essa pesquisa verificou que nenhum participante ultrapassou o índice de ingestão diária na quantidade consumida, mesmo entre aqueles que relataram consumir simultaneamente chás prontos e refrigerantes. Esses dados concordam com os reportados por Aguero et al. (2015), que observaram que nenhum estudante universitário que consumia bebidas contendo educolrantes ultrapassou a IDA estabelecida para cada edulcorante. Resultados semelhantes também foram reportados por Duran et al. (2011) sobre o consumo de edulcorantes artificiais com estudantes entre 6 e 14 anos em escolas na região de Valparaíso do Chile.

Observam-se na Tabela 4 os valores de ingestão diária aceitável para os edulcorantes presentes nas bebidas relatadas, considerando o peso corporal médio, máximo, mínimo e mediano dos participantes dessa pesquisa. Ainda nessa tabela, verifica-se em negrito a quantidade de copos $(200 \mathrm{~mL})$ que podem ser consumidos sem que o valor da IDA do edulcorante limitante presente na formulação da bebida seja ultrapassado. 
Tabela 4. Bebidas consumidas pelos participantes e seus respectivos consumos máximos para a média, máximo e mínimo e media do peso corpóreo dos participantes.

\begin{tabular}{|c|c|c|c|c|c|c|c|}
\hline \multirow[b]{2}{*}{ Produto } & \multirow[b]{2}{*}{ Edulcorante } & \multirow[b]{2}{*}{$\begin{array}{l}\text { Quantidade } \\
(\mathrm{mg} / 100 \mathrm{ml})\end{array}$} & \multirow[b]{2}{*}{$\begin{array}{c}\text { IDA } \\
\text { (mg/peso } \\
\text { corpóreo) }\end{array}$} & \multicolumn{4}{|c|}{ Consumo máximo: Copos de $200 \mathrm{ml}$} \\
\hline & & & & $\begin{array}{c}\text { Peso } \\
\text { Médio } \\
(75 \text { kg) }\end{array}$ & $\begin{array}{c}\text { Peso } \\
\text { Máximo } \\
(140 \text { kg) }\end{array}$ & $\begin{array}{c}\text { Peso } \\
\text { Mínimo } \\
\text { (41 kg) }\end{array}$ & $\begin{array}{r}\text { Mediana } \\
(90,5 \mathrm{~kg})\end{array}$ \\
\hline \multirow{3}{*}{ Coca-Cola zero } & Ciclamato de sódio & 27,0 & 11 & 15,3 & 28,5 & 8,4 & 18,4 \\
\hline & Aspartame & 12,0 & 40 & 125,0 & 233,3 & 68,3 & 150,8 \\
\hline & Acessulfame de Potássio & 15,0 & 15 & 37,5 & 70,0 & 20,5 & 45,3 \\
\hline \multirow{2}{*}{$\begin{array}{l}\text { Guaraná Antártica } \\
\text { Zero }\end{array}$} & Aspartame & 34,8 & 40 & 43,1 & 80,5 & 23,6 & 52,0 \\
\hline & Acessulfame de Potássio & 9,0 & 15 & 62,5 & 116,7 & 34,2 & 75,4 \\
\hline \multirow{2}{*}{ Pepsi Black } & Sucralose & 18,0 & 15 & $\mathbf{3 1 , 3}$ & 58,3 & 17,1 & 37,7 \\
\hline & Acessulfame de Potássio & 11,2 & 15 & 50,2 & 93,8 & 27,5 & 60,6 \\
\hline \multirow{2}{*}{ Sprite zero } & Ciclamato de sódio & 72,0 & 11 & 5,7 & 10,7 & 3,1 & 6,9 \\
\hline & Sacarina sódica & 11,0 & 5 & 17,0 & 31,8 & 9,3 & 20,6 \\
\hline \multirow{2}{*}{ Soda zero } & Acessulfame de Potássio & 9,0 & 15 & 62,5 & 116,7 & 34,2 & 75,4 \\
\hline & Aspartame & 34,8 & 40 & 43,1 & $\mathbf{8 0 , 5}$ & 23,6 & 52,0 \\
\hline \multirow{2}{*}{$\begin{array}{l}\text { Soda reduzido em 55\% de } \\
\text { açúcares }\end{array}$} & Acessulfame de Potássio & 6,2 & 15 & 351,6 & 656,3 & 192,2 & 424,2 \\
\hline & Sucralose & 1,6 & 15 & 90,7 & 169,4 & 49,6 & 109,5 \\
\hline \multirow{2}{*}{ Fanta Zero } & Ciclamato de sódio & 70,0 & 11 & 5,9 & 11,0 & 3,2 & 7,1 \\
\hline & Sacarina sódica & 8,0 & 5 & 23,4 & 43,8 & 12,8 & 28,3 \\
\hline \multirow{2}{*}{ Pepsi Twister zero } & Aspartame & 35,0 & 40 & 42,9 & 80,0 & 23,4 & 51,7 \\
\hline & Acessulfame de Potássio & 9.0 & 15 & 62,5 & 116,7 & 34,2 & 75,4 \\
\hline \multirow{2}{*}{$\mathrm{H} 20 \mathrm{H}$} & Aspartame & 31,5 & 40 & 47,2 & 88,9 & 26,0 & $\mathbf{5 7 , 5}$ \\
\hline & Acessulfame de Potássio & 8,1 & 15 & 69,4 & 129,6 & 38,0 & 83,8 \\
\hline \multirow{2}{*}{ Lipton ice zero açúcar } & Aspartame & 22,0 & 40 & 68,2 & 127,3 & $\mathbf{3 7 , 3}$ & 82,3 \\
\hline & Acessulfame de Potássio & 7,2 & 15 & 78,1 & 145,8 & 42,7 & 94,3 \\
\hline \multirow{3}{*}{ Ice Tea Leão } & Sacarina sódica & 13,0 & 5 & 14,4 & 26,9 & 7,9 & 17,4 \\
\hline & Acessulfame de Potássio & 6,0 & 15 & 93,8 & 175,0 & 51,3 & 113,1 \\
\hline & Sucralose & 5,0 & 15 & 112,5 & 210,0 & 61,5 & 135,8 \\
\hline
\end{tabular}

Fonte: Autores (2022).

No que se refere aos adoçantes de mesa líquidos (Figura 7), observa-se que a marca mais relatada pelos participantes foi a Linea com 34,15\% ( $n=28)$, seguida da marca Zero cal com 28,05\% ( $n=23)$. Observa-se também que a sucralose foi o edulcorante preferido pelos participantes, pois 35,37\% citaram as marcas Linea Sucralose, Zero Cal Sucralose e apenas sucralose sem identificação de marca. Vale ressaltar que independente do nome do adoçante ter um único edulcorante presente, não significa que a formulação desse produto será exclusivamente composta por esse edulcorante, como foi possível analisar após observação dos rótulos desses produtos. 
Figura 7. Marcas de adoçantes de mesa líquidos consumidos.

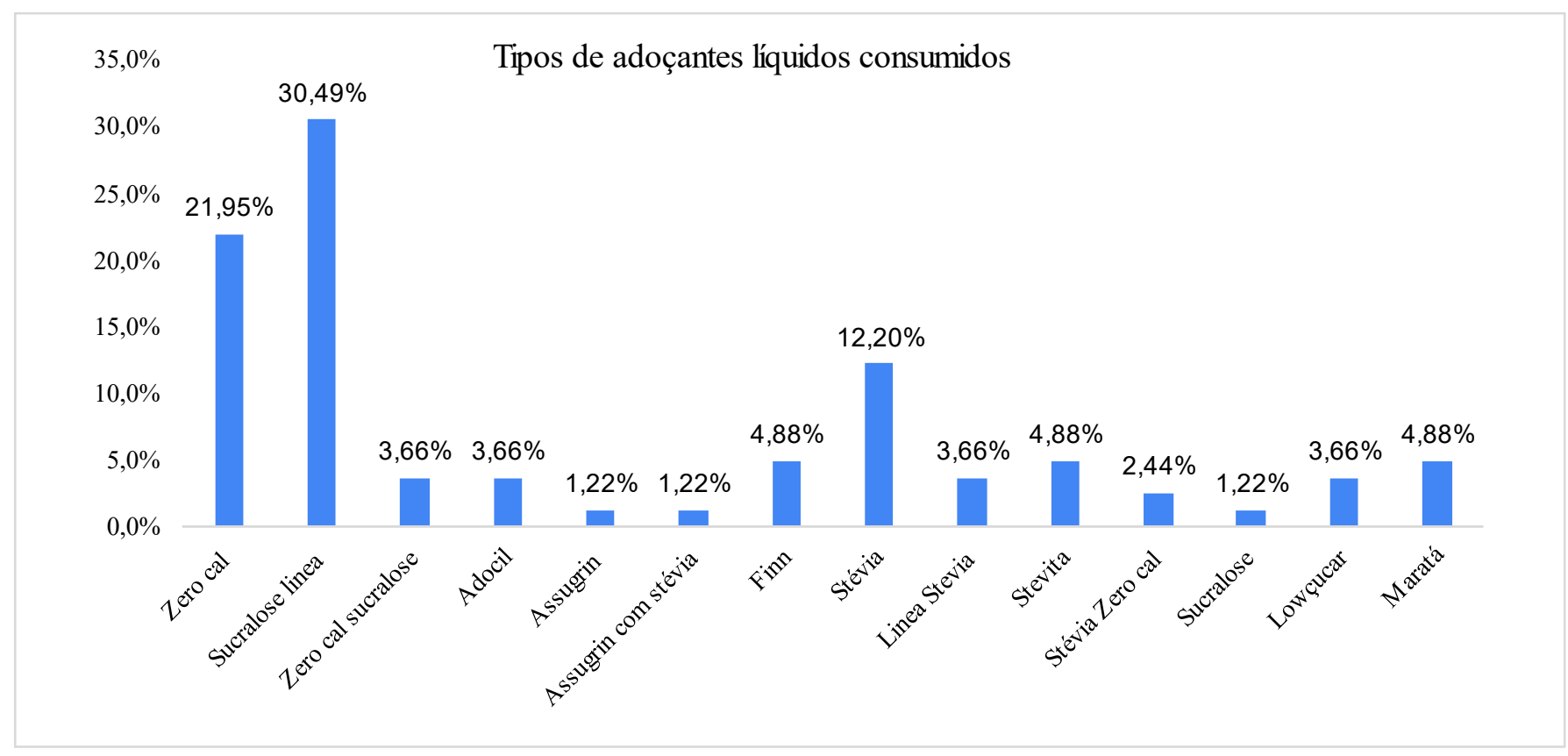

Fonte: Autores (2022).

Segundo Bian, et al. (2017), a sucralose é um dos adoçantes artificiais mais utilizados no mercado, devido ao seu sabor bastante açucarado, cerca de 600 vezes mais doce que a sacarose, e a ausência de retrogosto amargo, estabilidade em altas temperaturas e longa vida de prateleira. Oliveira, et al. (2019), ainda destacam a alta solubilidade em água desse edulcorante.

Lin, et al. (2021) também reportaram que a participação da sucralose no mercado vem aumentando em todo o mundo devido ao seu potencial adoçante e preço.

Ainda na Figura 7, edulcorantes compostos por stévia foram lembrados por $23,18 \%$ ( $\mathrm{n}=$ dos respondentes com as marcas Línea stévia, Stevita, Assugrin com Stévia, Stevia Zero Cal, e somente a Stévia. Por outro lado, há alguns anos Zanini, Araújo e Mesa- Martínez (2011) em Pelotas, Rio Grande do Sul, verificaram que apenas 3,3\% dos usuários referiram utilizar adoçante dietético à base de edulcorante natural (esteviosídeo). Dessa forma, pode-se concluir que os dados obtidos na presente pesquisa corroboram com as atuais tendências que indicam que os consumidores estão dispostos a experimentar alternativas naturais à sacarose e assim, o uso de adoçantes naturais pode representar uma grande oportunidade comercial para muitas empresas (Muñoz, et al., 2022).

Outras marcas também foram citadas, como Adocyl Zero Cal, Assugrin, Maratá e Lowçucar, entretanto, em função da não especificação da linha desses adoçantes por parte dos consumidores, não foi possível identificar a composição de edulcorantes presentes na formulação desses produtos.

Como dito anteriormente, os adoçantes líquidos de mesa, assim como os adoçantes em sachês, não têm a obrigatoriedade de informar a quantidade de cada edulcorante presente em sua formulação, o que impossibilita o cálculo dos valores da Ingestão Diária Aceitável desses produtos.

$\mathrm{Na}$ Tabela 5 são apresentados os adoçantes presentes na formulação das marcas citadas pelos fabricantes, exceto o adoçante Finn, devido à falta de especificação do tipo pelos participantes. 
Tabela 5. Marcas dos adoçantes de mesa líquidos consumidos pelos participantes e edulcorantes presentes.

\begin{tabular}{c|c}
\hline Marcas dos adoçantes & Adoçantes \\
\hline Sucralose linea & Sucralose e Acessulfame de potássio \\
\hline Zero cal sucralose & Sucralose e Acessulfame-K \\
\hline Assugrin & Ciclamato de Sódio, Sacarina Sódica e Acessulfame de Potássio \\
\hline Assugrin com Stévia & Ciclamato de sódio, sacarina sódica, acessulfame de potássio e \\
edulcorante natural: glicosídeos de esteviol
\end{tabular}

Fonte: Autores (2022).

Atualmente a legislação brasileira não obriga os adoçantes de mesa declarar a quantidade de cada edulcorante presente em suas formulações. Isso afeta o consumo de adoçantes naturais, por possuírem maior valor de mercado, visto que edulcorantes artificiais são mais baratos e possuem elevado sabor adoçante, reduzindo a quantidade de extrato natural necessário (Noronha, 2019). Portanto, a proporção de combinação entre esses adoçantes, pode ocasionar a redução do custo de produção e ainda assim manter o valor para produtos dessa categoria.

Verificou-se que $56 \%$ e $68 \%$ dos participantes utilizam entre 0-5 gotas de adoçante líquido para adoçar $50 \mathrm{~mL}$ de café e 200 mL de chá, respectivamente. Em relação aos sucos, a maioria (46\%) utiliza em média 6 gotas.

Apenas $20 \%$ dos participantes declararam utilizar adoçantes em sachês e as marcas descritas estão ilustradas na Figura 8. As mais utilizadas foram Zero Cal e Linea, assim como observado entre os adoçantes líquidos. Quanto a quantidade de sachê utilizada pelos participantes, $56 \%$ afirmou utilizar 1 sachê para adoçar cafés e chás, enquanto $73 \%$ também indicou 1 sachê para sucos. 
Figura 8. Marcas de adoçantes em sachês utilizados pelos participantes.

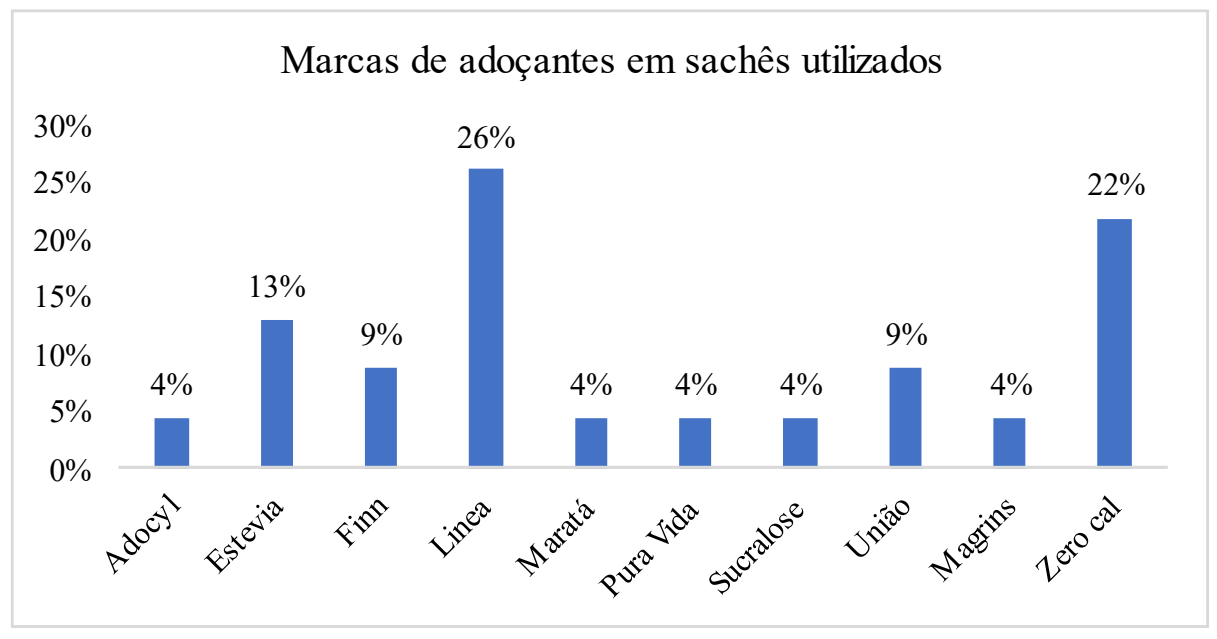

Fonte: Autores (2022).

O uso de adoçantes não nutritivos vem aumentando como alternativa para a substituição aos açúcares presentes nas formulações dos alimentos, mas dados sobre frequência de uso e níveis de consumo ainda são escassos (Vaesken et al.,2020) e tendo em vista que todos os edulcorantes regulamentados pela legislação são seguros dentro do limite de consumo diário indicado para cada um (Oliveira, et al., 2019), estudos relacionados à frequência de consumo para determinar o IDA da população se tornam cada vez mais relevantes.

Dentre as limitações encontradas durante a execução da pesquisa, destaca-se a complexibilidade em obter com precisão a frequência de consumo pelos participantes, visto a dificuldade em estabelecer um padrão de periodicidade de ingestão dos entrevistados em uma pesquisa tão aberta como essa, além da ausência da porcentagem dos edulcorantes contidos na formulação dos adoçantes de mesa, impedindo o cálculo da IDA desses produtos.

\section{Conclusão}

Verificou-se que o principal motivo do consumo de produtos diet/light e adoçantes de mesa líquidos e sachês é a preocupação com a saúde, $51 \%$ alegaram que conheciam o adoçante presente nos produtos consumidos e que o consumo de produtos diet/light foi concentrado em participantes com maior escolaridade, renda familiar e do sexo feminino. Pelo cálculo da Ingestão Diária Aceitável foi verificado que nenhum participante atingiu seu respectivo IDA pelo edulcorante determinante no produto consumido. Os adoçantes de mesa possuem alta frequência de consumo pelos participantes, entretanto, no rótulo dos adoçantes de mesa líquido não são apresentadas a proporção de cada edulcorante na formulação, assim como os rótulos de adoçantes em sachês, impedindo o cálculo de índice de ingestão diária. Também podemos concluir o aumento na tendência na procura de produtos com adoçantes naturais e o aumento da informação por parte dos consumidores.

Sugere-se para trabalhos futuros o aumento do número de participantes, a fim de se obter maior representativa do consumo de edulcorantes pela população brasileira, além de aprimorar a coleta de dados relacionados à quantidade e frequência, dessa forma propõe-se alteração na metodologia, onde os participantes deverão registrar diariamente, durante determinado intervalo de tempo, todos os produtos diet e light consumidos, bem como suas respectivas quantidades, e dessa forma determinar o índice de ingestão diária com maior confiabilidade. 


\section{Referências}

Aguero, D., S., Cornwall, R., J., Vegas, E., C., Ariza, S., J., S., Arrivillagas, C., K, Bujaicos, C., P., M., Alzamora, A., S., \& Bernados, E, S. (2015). Consumo de edulcorantes no nutritivos en bebidas carbonatadas en estudiantes universitarios de algunos países de Latinoamérica. Nutrición Hospitalaria, 31(2), 959965. 10.3305/nh.2015.31.2.8026

Barros, S. P, Ferreira, V. T. \& Pereira, J. R. (2021). Consumo de edulcorantes artificiais por gestantes e nutrizes de Tocantins. Revista Desafios, 8, 80-84. doi.org/10.20873/uftsupl2021-12504

Bian, X., Chi, L., Gao, B., Tu, P., Ru, H., \& Lu, K. Gut Microbiome Response to Sucralose and Its Potential Role in Inducing Liver Inflammation in Mice. Frontiers in Physiology, 8 (487). doi.org/10.3389/fphys.2017.00487

Bianchi, D, M. (2012). Avaliação dos níveis de ingestão diária de edulcorantes pelo consumo de adoçantes líquidos de mesa. Dissertação Mestrado em Engenharia de Alimentos. Universidade Estadual de Campinas, Campinas, São Paulo, Brasil.

Brasil. Ministério da Saúde. Agência Nacional de Vigilância Sanitária. Resolução de Diretoria Colegiada - RDC nº 18 , de 24 de março de 2008 . Regulamento Técnico que autoriza o uso de aditivos edulcorantes em alimentos, com seus respectivos limites máximos. 2008. https://www.gov.br/agricultura/ptbr/assuntos/inspecao/produtos-vegetal/legislacao-1/biblioteca-de-normas-vinhos-e-bebidas/resolucao-rdc-no-18-de-24-de-marco-de-2008.pdf/view

Durán, A, S., Quijada, M., M., Silva, V., L Almonacid, M., N., Berlanga, Z., M., \& Rodrigues, N., M (2011). Niveles de ingesta diaria de edulcorantes no nutritivos en escolares de la región de Valparaíso. Rev Chil Nutr 38 (4), 444-449.

Duran, A., S., Cordon, K., A., \& Rodrigues, N, P, M. (2013) Edulcorantes no nutritivos, riesgos, apetito y ganancia de peso. Rev Chil Nutr, 8 (3).

Hernandez, B., Frias, V., R., Abreuc, A., A., T., Valdez., A., P., Nava, B., L., A., Sancheszf, C., R., I., Saenzg., C., J., Sanchez, C., A., Flores, E., A., J., Rosiles, H., V., Hernández-Vez, G., Chávez, I., M., E., Lugoi., N., A., Romo-Romo, A., Margaín, R., A., Díaz, V., M., A., \& Mondragón, Z., F., E., (2019). Review of the scientific evidence and technical opinion on noncaloric sweetener consumption in gastrointestinal diseases. Revista de Gastroenterología de México, 84(4), 492-510. doi.org/10.1016/j.rgmxen.2019.08.001

Honorio, R., A, Soares, F., A., Lima, N., C., D., \& Tribst, L., A., A. Passion fruit nectar sweetened with stevia and sucralose: Is perception affected by the regular consumption of sweeteners or diabetes? International Journal of Gastronomy and Food Science. 25, 1- 10. doi.org/10.1016/j.ijgfs.2021.100404.

Joint Expert Committee on Food Additives (JECFA), (2019). Edición en línea: Compendio de especificaciones para aditivos alimentarios.: https://www.fao.org/food/food-safety-quality/scientific-advice/jecfa/jecfa-additives/es/.

Li, D., O’Brien, W., J., Tscharke, J., B., Choi, M., P., Ahmed, F., Thompson, J., Mueller, F., J., Sun, H., \& Thomas, V, K. (2021). Trends in artificial sweetener consumption: A 7-year wastewater-based epidemiology study in Queensland, Australia. Clinical Nutrition ESPEN, 754. doi.org/10.1016/j.scitotenv.2020.142438

Lima, C., R. (2020). Avaliação dos conflitos de interesses financeiros em publicações sobre o consumo de edulcorantes de alta intensidade e alterações do peso corporal: uma revisão sistemática. Dissertação Mestrado em Ciências Cardiovasculares. Instituto Nacional de Cardiologia, Rio de Janeiro, Rio de Janeiro, Brasil.

Lopes, C., G., Lima., S., V., Mendonça, T., T., Oliveira, P., K., S., Pereira, P., C., Bezerra, N., A., \& Albuquerque, V., N. (2020). Consumo de adoçantes por pacientes com diabetes mellitus tipo 2: uma revisão integrativa. Research, Society and Development, 9 (10), 1- 22. dx.doi.org/10.33448/rsd-v9i10.8619

Muñoz, C., R., Delgado., C., M., Almeida., C., R., Nava., L., D., Chavez- Muñoz, M., Velásquez-Chávez, F., V., Torres-Hernandez, E., C, Castro, G., E., \& Ahmad, Z., M., (2022). Natural sweeteners: Sources, extraction and current uses in foods and food industries. Food Chemistry, 370. doi.org/10.1016/j.foodchem.2021.130991

Noronha, C., P., F., I. (2019). Determinação de edulcorantes e constituintes inorgânicos em adoçantes de mesa. Dissertação Mestrado em Engenharia Química. Universidade Federal de Minas Gerais, Belo Horizonte, Minas Gerais, Brasil.

Oliveira, R., L., Souza, L., V., P., Santos, M., G., \& Barros, A., V. (2019). Avaliação dos edulcorantes presentes em produtos diet. Revista Brasileira de Obesidade, Nutrição e Emagrecimento, 8, 498-507.

Sylvetsky, C., A., \& Rother, K. (2016). Trends in the consumption o flow-caloriesweeteners. Physiology \& Behavior, 164. doi.org/10.1016/j.physbeh.2016.03.030

Vaesken, S., L., M., Partearroyo, T., \& Moreiras, V. (2020). Edulcorantes bajos en calorías o sin calorías, dieta y salud: una visión actual. Nutrición Hospitalaria, 37, 24-27. doi.org/10.20960/nh.03352

Zanini, V., R., Araújo, L., C., \& Mesa-Martinez (2011). Utilização de adoçantes dietéticos entre adultos em Pelotas, Rio Grande do Sul, Brasil: um estudo de base populacional. Cad. Saúde Pública, 27 (5), 924-934. doi.org/10.1590/S0102-311X2011000500010 